\title{
Sulla generalizzazione delle frazioni continue algebriche.
}

\author{
(Di S. Pincherle, a Bologna.)
}

In una breve Nota pubblicata nel 1889 nei Rendiconti dell' Istituto Lombardo $\left(^{*}\right)$ e poi in una Memoria presentata alla $R$. Accademia delle Scienze di Bologna $\left({ }^{*}\right)$ ho indicato in qual modo, partendo da due o più funzioni date, sia possibile definire per esse un algoritmo analogo a ciò che è, per il caso di una sola funzione, lo sviluppo in frazione continua. Esso si genera quando si vogliono legare le due funzioni date $\sigma_{1}, \sigma_{2}$ mediante una relazione lineare a coefficienti razionali :

$$
A \sigma_{1}+B \sigma_{2}+C=0
$$

nel modo il più approssimato possibile per un dato grado dei coefficienti $A, B, C$.

Nel presente scritto espongo alcune nuove proprietà di cui gode quest'algoritmo, ed alcune applicazioni cui esso si presta. Per semplicità, mi limito al primo stadio della generalizzazione e ciò̀ al caso che si parta da due funzioni date, nel quale caso la base della teoria è una relazione ricorrente a quattro termini; avvertendo però che presenterebbe lievi difficoltà, e di pura forma, l'estensione al caso generale in cui si parte da $p$ funzioni date ed in cui la teoria si fonda sopra una relazione ricorrente a $p+2$ termini.

Le proposizioni dei $\S \S 1-4,6$ e 8 della presente Memoria sono contenute come casi particolari nei teoremi dati nella citata Memoria dell'Accademia di Bologna del marzo 1890.

(*) Serie II, tomo 22 , fasc. 12.13.

(\%) Serie IV, tomo 10, marzo 1890. 
$\grave{E}$ dovere gradito per me il cogliere questa occasione per esprimere la

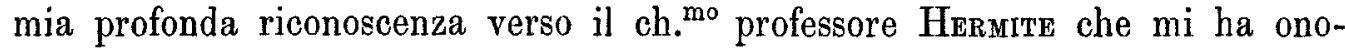
rato, a proposito di queste ricerche, di preziosi e benevoli consigli. Occupatosi egli pure di ricerche consimili, l'illustre uomo è giunto dal canto suo ad un algoritmo che presenta con quello da me trovato la più grande analogia, e contemporaneamente, ed indipendentemente l'uno dall' altro, abbiamo trovato che l'algoritmo che serve a legare nel modo più approssimato possibile due funzioni $\sigma_{1}, \sigma_{2}$ mediante una relazione lineare a coefficienti razionali, è anche quello che serve a rappresentare nel modo più approssimato possibile le due funzioni $\sigma_{1}, \sigma_{2}$ mediante frazioni razionali di uguale denominatore. (Vedi $\S 9$ di questa Memoria.) Dalle comunicazioni epistolari da lui gentilmente favoriterni, posso aggiungere che l'HERMIтE lega queste sue ricerche a questioni interessanti di calcolo integrale, in ispecie a quelle profonde investigazioni sulle funzioni esponenziali che hanno servito di fondamento al suo celebre lavoro sulla irrazionalità della funzione $e^{z}$; ho detto quanto basta perchè debba essere nel desiderio di tutti che i risultati ottenuti in questo campo dall' esimio analista vengano presto alla luce $\left({ }^{*}\right)$.

1. Siano:

$$
\sigma_{1}=\sum_{n=1}^{\infty} \frac{s_{n}}{x^{n}}, \quad \sigma_{2}=\sum_{n=2}^{\infty} \frac{s_{n}^{\prime}}{x^{n}},
$$

due serie di potenze decrescenti di $x ; s_{1}$ ed $s_{2}$ siano essenzialmente diversi da zero, per modo che $\sigma_{1}$ sia del grado -1 e $\sigma_{2}$ del grado -2 . Pongo:

$$
1+a_{0} \sigma_{1}+b_{0} \sigma_{2}=\sigma_{3} ;
$$

posso sempre determinare il binomio di primo grado

$$
a_{0}=a_{0}^{\prime} x+a^{\prime \prime}{ }_{0},
$$

e la costante $b_{0}$ in guisa che $\mathrm{i}$ termini in $x^{2}, x^{-1}, x^{-2}$ spariscano dalla (2); la $\sigma_{3}$ si riduce allora al grado -3 . Essa potrebbe anche ridursi ad un grado inferiore, se i coefficienti delle serie (1) fossero legati da certe relazioni, di

(*) La generalizzazione delle frazioni continue intrapresa nel furo campo aritmetico dal $\mathfrak{J}_{\mathrm{ACOBI}}$ (Vedi Memoria postuma pubblicata dall'HeINe, J. de Crelle, tomo 60) sembra doruta ad ispirazione dell' Henmite stesso. 
cui la prima sarebbe:

$$
\begin{array}{lll}
s_{2} & s_{1} & 0 \\
s_{3} & s_{2} & s_{2}^{\prime} \\
s_{4} & s_{3} & s_{3}^{\prime}
\end{array}=0 ;
$$

ma escludo questo caso eccezionale. Pongo in seguito:

$$
\sigma_{1}+a_{1} \sigma_{2}+b_{1} \sigma_{3}=\sigma_{4}
$$

e qui determino, il che è sempre possibile, il binomio di primo grado $a_{1} \mathrm{e}$ la costante $b_{1}$ in guisa che $\mathrm{i}$ termini in $x^{-1}, x^{-2}, x^{-3}$ scompaiono; $\sigma_{4}$ è allora di grado -4 , e si escluda il caso eccezionale che possa essere di grado inferiore.

Proseguendo in questo modo, si viene a costruire lo schema

$$
\left\{\begin{array}{ccc}
1 & a_{0} & b_{0} \\
1 & a_{1} & b_{1} \\
1 & a_{2} & b_{2} \\
\ldots & \ldots \\
1 & a_{n} & b_{n} \\
\ldots & \ldots
\end{array}\right\}
$$

che dico ottenuto mediante l'algoritmo generalizzato delle frazioni continue algebriche. L'algoritmo si dirà normale nel caso in cui mi sono posto, che $\sigma_{n}$ sia esattamente del grado $-n$ (e non di grado inferiore): in tal caso le $a_{n}$ sono binomii lineari

$$
a_{n}=a_{n} x+a^{\prime \prime},
$$

e le $b_{n}$ sono costanti; in caso diverso, le $a_{n}$ e $b_{n}$ potrebbero essere polinomi di grado superiore.

2. Lo schema (3) ci rappresenta il sistema di equazioni:

$$
\begin{aligned}
& 1+a_{0} \sigma_{1}+b_{0} \sigma_{2}=\sigma_{3} \\
& \sigma_{1}+a_{1} \sigma_{2}+b_{1} \sigma_{3}=\sigma_{1} \\
& \ldots \ldots \\
& \sigma_{n}+a_{n} \sigma_{n+1}+b_{n} \sigma_{n+2}=\sigma_{n+3} \\
& \ldots \ldots \ldots
\end{aligned}
$$


Eliminando fra queste le $\sigma_{3}, \sigma_{4}, \ldots \sigma_{n+2}$, si ottiene la relazione:

$$
A_{n+3}+B_{n+3} \sigma_{1}+C_{n+3} \sigma_{2}=\sigma_{n+3}
$$

dove $A_{n+3}, B_{n+3}, C_{n+3}$ sono polinomii razionali interi in $x$ la cui considerazione è importantissima per ciò che segue.

3. Anzitutto, sostituendo per $\sigma_{n+3}, \sigma_{n+2}, \sigma_{n+1}, \sigma_{n}$ le loro espressioni della forma (5) nella $n+1 .^{\mathrm{a}}$ delle (4), viene:

$$
\begin{gathered}
A_{n}+a_{n} A_{n+1}+b_{n} A_{n+2}-A_{n+3}+\sigma_{1}\left(B_{n}+a_{n} B_{n+1}+b_{n} B_{n+2}-B_{n+3}\right) \\
+\sigma_{2}\left(C_{n}+a_{n} C_{n+1}+b_{n} C_{n+2}-C_{n+2}\right)=0
\end{gathered}
$$

ma questa uguaglianza si deduce dallo specchio di relazioni (4) in modo puramente formale, cioè qualunque siano le quantità $\sigma_{1}$ e $\sigma_{2}$. Deve adunque essere:

$$
\begin{aligned}
& A_{n}+a_{n} 1_{n+1}+b_{n} A_{n+2}=A_{n+3}, \\
& B_{n}+a_{n} B_{n+1}+b_{n} B_{n+2}=B_{n+3}, \\
& C_{n}+a_{n} C_{n+1}+b_{n} C_{n+2}=C_{n+3},
\end{aligned}
$$

donde segue che ${ }_{1}{ }_{n}, B_{n}, C_{n}$ costituiscono, come pure $\sigma_{n}$, altrettanti integrali dell'equazione lineare, alle differenze:

$$
f(n+3)-b_{n} f(n+2)-a_{n} f(n+1)-f(n)=0 .
$$

Si dà uno specchio dei valori iniziali dei polinomii $A_{n}, B_{n}, C_{n}$ :

$$
\begin{aligned}
& A_{0}=1, \ldots \ldots \ldots \quad B_{0}=0, \ldots \ldots \ldots, C_{0}=0, \\
& \Lambda_{1}=0, \ldots \ldots \ldots \ldots \ldots C_{1}=0, \\
& A_{2}=0, \ldots \ldots \ldots, B_{2}=0, \ldots \ldots \ldots \ldots C_{2}=1 \\
& A_{3}=1, \ldots \ldots \quad B_{3}=a_{0}, \ldots \ldots \ldots \ldots \quad C_{3}=b_{0}, \\
& \Lambda_{1}=b_{1}, \ldots \ldots \quad B_{4}=1+a_{0} b_{1}, \ldots \ldots \ldots \quad C_{4}=a_{1}+b_{0} b_{1} \text {, } \\
& ._{5}=a_{2}+b_{1} b_{2}, \ldots \quad B_{5}=b_{0}+a_{0} a_{2}+a_{0} b_{1} b_{2}, \ldots \quad C_{5}=1+a_{2} b_{0}+a_{1} b_{2}+b_{0} b_{1} b_{2} .
\end{aligned}
$$

ecc.

I valori di $1_{n}, B_{n}, C_{n}$ per $n=0,1,2$ mostrano che questi polinomii formano un sistema fondamentale d'integrali della equazione (7). 
Dalle relazioni (6) si stabilisce facilmente il grado dei polinomii $A_{n}, B_{n}$, $C_{n}$. Essi sono dati dal seguente quadro:

\begin{tabular}{|c|c|c|}
\hline Gradi di & per $n=2 r$ & per $n=2 r+1$ \\
$A_{n}$ & $r-2$ & $r-1$ \\
$B_{n}$ & $r-1$ & $r$ \\
$C_{n}$ & $r-1$ & $r-1$ \\
\hline
\end{tabular}

4. Se ora date le serie $\sigma_{1}, \sigma_{2}$, si proponesse di determinare $i$ polinomii $A, B, C$ del grado indicato dallo specchio precedente in modo che la relazione:

$$
1+B \sigma_{1}+C \sigma_{2}=0
$$

sia verificata fino ai termini in $x^{-n+1}$ inclusivamente, si tioverebbe senza difficoltà che $1, B, C$ non possono differire da $\Lambda_{n}, B_{n}, C_{n}$ che per un fattore costante. Il nostro algoritmo serve dunque a risolvcre il

Problema I. Date due funzioni, stalitive fra esse una relazione lineare a coefficienti razionali la più approssimata possibile per un grado dato dei coefficienti.

5. Non è privo d'interesse il vedere come siano formati i coefficienti della massima potenza di $x$ nei polinomii $A_{n}, B_{n}, C_{n}$. Indichiamo rispettivamente questi coefficienti con $g_{n}, h_{n}, k_{n}$. Nella relazione ricorrente (6), cioc̀:

$$
A_{n+3}=\Lambda_{n}+a_{n} A_{n+1}+b_{n} \Lambda_{n+2},
$$

si ha, per $n=2 r$, che $1_{n}$ è di grado $r-2$ ed $A_{n+1}$ e $A_{n+2}$ di grado $r-1$, mentre $A_{n+3}$ è di grado $r$; e siccome

$$
a_{n}=a_{n}^{\prime} x+a_{n}^{\prime \prime},
$$

non vi ̀̀ che un termine di grado $r$ nel secondo membro, proveniente da $\iota_{n} A_{n+1}$. Si ha dunque:

$$
g_{2 r+3}=a_{2 r}^{\prime} g_{2 r+1} \text {. }
$$

Invece, per $n=2 r+1$, si ha $A_{n}$ del grado $r-1, A_{n+1}$ del grado $r-1$, $l_{n+2}$ ed $A_{n+3}$ del grado $r$. Vi sono dunque due termini di grado $r$ nel se- 
condo membro, l'uno proveniente da $A_{n+2}$, l'altro da $a_{n} A_{n+1}$, onde

$$
g_{2 r+1}=a_{2 r+1}^{\prime} g_{2 r+2}+b_{2 r+1} g_{2 r+3} \text {. }
$$

Analogamente si trova:

$$
\begin{aligned}
& h_{2 r+3}=a_{2 r}^{\prime} h_{2 r+1}, \\
& h_{2 r+4}=a_{2 r+1}^{\prime} h_{2 r+2}+b_{2 r+1} h_{2 r+3} . \\
& k_{2 r+3}=a_{2 r}^{\prime} k_{2 r+1}+b_{2 r} k_{2 r+2}, \\
& k_{2 r+4}=a_{2 r+4}^{\prime} k_{2 r+2} .
\end{aligned}
$$

Da queste formole si deducono le seguenti espressioni, che sembrano abbastanza degne di nota:

$$
\left.\begin{array}{l}
g_{2 r+1}=a_{2}^{\prime} a_{4}^{\prime} a_{6}^{\prime} \ldots a_{2 r-2}^{\prime}, \\
h_{2 r+1}=a_{0}^{\prime} a_{2}^{\prime} a_{1}^{\prime} \ldots a_{2 r-2}^{\prime}, \\
k_{2 r+2}=a_{1}^{\prime} a_{3}^{\prime} a_{5}^{\prime} \ldots a_{2 r-1}^{\prime} .
\end{array}\right\}
$$

6. Se si forma il determinante

$$
\left(A_{n+1} B_{n+2} C_{n+3}\right)=\begin{array}{lll}
A_{n+1} & B_{n+1} & C_{n+1} \\
A_{n+2} & B_{n+2} & C_{n\llcorner 2} \\
A_{n+3} & B_{n+3} & C_{n+3}^{\prime}
\end{array},
$$

poi si snstituiscono agli elementi dell' ultima linea le loro espressioni tolte dalle $(6)$, si trova senz'altro che il determinante stesso è uguale ad $\left(A_{n} B_{n+1} C_{n+2}\right)$, e per conseguenza ad $\left(A_{0} B_{1} C_{2}\right)$, il quale è uguale all' unità. Onde si ha, qualunque sia $n$ :

$$
\left(A_{n} B_{n+1} C_{n+2}\right)=1 \text {. }
$$

7. Ci conviene ora di studiare $\mathrm{i}$ polinomii:

$$
\begin{aligned}
& P_{n}=B_{n} C_{n+1}-C_{n} B_{n+1}, \\
& Q_{n}=C_{n} A_{n+1}-A_{n} C_{n+1}, \\
& R_{n}=A_{n} B_{n+1}-B_{n} A_{n+1} .
\end{aligned}
$$

Si ha dapprima, dalle (15):

$$
\begin{aligned}
& A_{n} P_{n}+B_{n} Q_{n}+C_{n} R_{n}=0, \\
& A_{n+1} P_{n}+B_{n+1} Q_{n}+C_{n+1} R_{n}=0, \\
& A_{n+2} P_{n}+B_{n+2} Q_{n}+C_{n+2} R_{n}=1 ;
\end{aligned}
$$


onde risulta che:

$$
\left.\begin{array}{l}
A_{n}=Q_{n} R_{n-1}-R_{n} Q_{n-1} \\
B_{n}=R_{n} P_{n-1}-P_{n} R_{n-1} \\
C_{n}=P_{n} Q_{n-1}-Q_{n} P_{n-1} .
\end{array}\right\}
$$

Si determina poi facilmente il grado dei polinomii $P_{n}, Q_{n}, R_{n}$, pel mezzo delle formole (16) e dello specchio (8) dato dianzi, e si trova che il grado di $P_{n}$ è $n-1$ al più, ed il grado di $Q_{n}$ e di $R_{n}$ è al più $n-2$. Ma dalle proprietà dei coefficienti dei termini di più alto grado in $x$ nelle $1_{n}, B_{n}$ e $C_{n}$, proprictà date al $\S 5$, si trova con un calcolo dei più elementari che il coefficiente di $x^{n-1}$ in $P_{n}$ e di $x^{n-2}$ in $Q_{n}$ è diverso da zero, mentre il coefficiente di $x^{n-2}$ in $R_{n}$ è nullo, ma non quello di $x^{n-3}$. Donde risulta che i gradi di $P_{n}, Q_{n}, R_{n}$ sono rispettivamente $n-1, n-2$ ed $n-3$.

8. I polinomii $P_{n}, Q_{n}, R_{n}$ sono integrali dell' equazione alle differenze:

$$
f(n-1)-b_{n-1} f(n)-a_{n} f(n+1)-f(n+2)=0
$$

a dimostrare ciò, basta riprendere le formole del $\S 3$ :

$$
\begin{aligned}
& C_{n+3}=b_{n} C_{n+2}+a_{n} C_{n+1}+C_{n}, \\
& B_{n+3}=b_{n} B_{n+2}+a_{n} B_{n+1}+B_{n},
\end{aligned}
$$

moltiplicare la prima per $B_{n+1}$, la seconda per $C_{n+1}$ e sottrarre, si ottiene in tal $\operatorname{modo}$ :

$$
P_{n+2}=-a_{n} P_{n+1}+C_{n} B_{n+2}-B_{n} C_{n+2} .
$$

Ma analogamente si ha:

$$
C_{n} B_{n+2}-B_{n} C_{n+2}=-b_{n+1} P_{n}+P_{n-1}^{\prime}
$$

onde sostituendo:

$$
P_{n+2}=-a_{n} P_{n+1}-b_{n-1} P_{n}+P_{n-1}
$$

c. d. d.

Il teorema si dimostra nel modo stesso per $Q_{n}$ e per $R_{n}$.

Osservazione. In varie ricerche sulle equazioni lineari alle differenze, accanto ad un'equazione della forma:

$$
a_{n} f(n+m)+b_{n} f(n+m-1)+\cdots+h_{n} f(n)=0,
$$


si presenta spontanea la considerazione dell'equazione:

$$
a_{n-m} f(n-m)+b_{n-m+1} f(n-m+1)+\cdots+h_{n} f(n)=0 ;
$$

questa seconda equazione l'ho chiamata inversa $\left({ }^{*}\right)$ della precedente. L'equazione alle differenze cui soddisfano $P_{n}, Q_{n}, R_{n}$ è appunto l'inversa di quelle cui soddisfano $A_{n}, B_{n}, C_{n}$.

L'equazione ricorrente (18), insieme al sistema dei valori iniziali

$$
\begin{array}{lll}
P_{1}=0, & Q_{1}=0, & R_{1}=1, \\
P_{2}=1, & Q_{2}=0, & R_{2}=0 \\
P_{3}=-a_{0}, & Q_{3}=1, & R_{3}=0,
\end{array}
$$

serve a determinare completamente i polinomii $P_{n}, Q_{n}, R_{n}$.

Dallo specchio precedente di valori iniziali risulta ancora che le $P_{n}, Q_{n}$, $R_{n}$ costituiscono un sistema fondamentale d'integrali dell' equazione (18).

9. Riprendiamo le relazioni lineari della forma (5) fra $\sigma_{n}, \sigma_{n+1}$ e $\sigma_{1}, \sigma_{2}$; esse sono:

$$
\begin{aligned}
& \sigma_{n+1}=A_{n+1}+B_{n+1} \sigma_{1}+C_{n+1} \sigma_{2}, \\
& \sigma_{n}=A_{n}+B_{n} \sigma_{1}+C_{n} \sigma_{2} .
\end{aligned}
$$

Risolvendo rispetto a $\sigma_{1}, \sigma_{2}$, se ne ricava:

$$
\begin{aligned}
& \sigma_{1}=\frac{Q_{n}+C_{n+1} \sigma_{n}-C_{n}{ }_{n+1},}{P_{n}}, \\
& \sigma_{2}=\frac{R_{n}+B_{n} \sigma_{n+1}-B_{n+1}-n}{P_{n}},
\end{aligned}
$$

e ponendo:

$$
\lambda_{n}=C_{n+1} \sigma_{n}-C_{n} \sigma_{n+1}, \quad \mu_{n}=B_{n} \sigma_{n+1}-B_{n+1} \sigma_{n},
$$

si ha:

$$
\left.\begin{array}{l}
\sigma_{1}-\frac{Q_{n}}{P_{n}}=\frac{\lambda_{n}}{P_{n}} \\
\sigma_{2}-\frac{R_{n}}{P_{n}}=\frac{\mu_{n}}{P_{n}}
\end{array}\right\}
$$

(*) Vedi per es. Compte-Rendus de l'Académie des Sciences, tome 107, pag. 986. Paris, 17 décémbre 1888 . 
I gradi di $\lambda_{n}, \mu_{n}$ si calcolano senza difficoltà, e si trova che:

$$
\begin{aligned}
\text { per } n=2 r \quad \text { il grado di } \lambda_{n} \text { è }-r-1 \text { e quello di } \mu_{n} \grave{\mathrm{e}}-r, \\
\eta n=2 r+1 \quad \eta \quad \lambda_{n} \geqslant-r-1 \quad \eta \quad \mu_{n} n-r-1 .
\end{aligned}
$$

Ne consegue che le frazioni $\frac{Q_{n}}{P_{n}}, \frac{R_{n}}{P_{n}}$ sviluppate in serie di potenze intere negative di $x$ coincidono rispettivamente con $\sigma_{1}$ e $\sigma_{2}$ fino ai termini di grado

$$
\begin{array}{llll}
-3 r+1 & \text { e }-3 r+2 & \text { per } & n=2 r \\
-3 r & n-3 r & \text { n } & n=2 r+1
\end{array}
$$

inclusivamente.

Se ora ci si propone, date le serie $\sigma_{1}, \sigma_{2}$, di determinare due frazioni razionali aventi lo stesso denominatore ed il cui sviluppo in serie di potenze decrescenti coincida con $\sigma_{1}, \sigma_{2}$ fino ad un grado dato (lo stesso per le due frazioni o differente al più di un' unità) ed il massimo possibile compatibilmente col grado dei termini delle due frazioni, si trova facilmente, col metodo dei coefficienti indeterminati, che esse non possono differire da $\frac{Q_{n}}{P_{n}}, \frac{R_{n}}{P_{n}}$. Il nosiro algoritmo serve dunque a risolvere il

Problema II. Date due funzioni, rappresentarle mediante due frazioni razionali aventi il medesimo denominatore nel modo pii approssimato possibile compatibilmente col grado dato del denominatore di queste frazioni.

Osservazione. Le (20), che si possono scrivere:

$$
P_{n} \sigma_{1}-Q_{n}=\lambda_{n}, \quad P_{n} \sigma_{2}-R_{n}=\mu_{n},
$$

mostrano che $\lambda_{n}, \mu_{n}$ sono anch'esse integrali dell' equazione ricorrente (18).

10. Le seguenti formole fanno risaltare sempre più l' analogia fra il nostro algoritmo e le frazioni continue algebriche. Si ricava infatti dalle (17):

$$
\frac{Q_{n}}{P_{n}^{-}}-\frac{Q_{n-1}}{P_{n-1}}=-\frac{C_{n}}{P_{n} P_{n-1}}, \quad \frac{R_{n}}{P_{n}}-\frac{R_{n-1}}{P_{n-1}}=\frac{B_{n}}{P_{n} P_{n-1}},
$$

dove i secondi membri sono rispettivamente dei gradi:

$$
\begin{array}{llll}
-3 r+2, & -3 r+2 & \text { se } & n=2 r \\
-3 r, & -3 r+1 & & n
\end{array}
$$


Ne segue:

$$
\begin{aligned}
& \frac{Q_{n}}{P_{n}}=-\frac{C_{2}}{P_{1} P_{2}}-\frac{C_{3}}{P_{2} P_{3}}-\cdots-\frac{C_{n}}{P_{n-1} P_{n}}, \\
& \frac{R_{n}}{P_{n}}=\frac{B_{3}}{P_{2} P_{3}}+\frac{B_{4}}{P_{3} P_{1}}+\cdots+\frac{B_{n}}{P_{n-1} P_{n}} \cdot
\end{aligned}
$$

Si può dunque scrivere:

$$
\sigma_{1}=-\sum_{n=1}^{\infty} \frac{C_{n+1}}{P_{n} P_{n+1}}, \quad \sigma_{2}=\sum_{n=2}^{\infty} \frac{R_{n+1}}{P_{n} P_{n+1}} .
$$

In queste formule, come in quelle analoghe della teoria delle frazioni continue, la convergenza delle serie non è dimostrata e dovrà verificarsi volta per volta. L'uguaglianza fra $\sigma_{1}, \sigma_{2}$ e le serie dei secondi membri va intesa in questo senso: che prendendo in esse serie un numero di termini sempre crescente e sviluppando in serje di potenze negative di $x$, vi sarà un numero sempre maggiore di termini che andrà coincidendo coi termini di $\sigma_{1}$ e $\sigma_{2}$.

11. Pongasi ora:

$$
\sigma_{1}=\int_{l} \frac{p(y) d y}{x-y}, \quad \sigma_{2}=\int_{l_{1}} \frac{o_{1}(y) d y}{x-y},
$$

coll' ipotesi :

$$
\int_{l_{1}} \varphi_{1}(y) d y=0
$$

e dove l'integrazione va estesa alle linee $l, l_{1}$ di lunghezza finita e per altro qualunque (aperte o chiuse) nel piaz. della variabile $y$. Si deduce dalle (21):

$$
\begin{aligned}
& \int_{i} \frac{P_{n}(x)-P_{n}(y)}{x-y} \varphi(y) d y+\int_{l} \frac{P_{u}(y) \cdots(y) d y}{x-y}=Q_{n}+i_{n}, \\
& \int_{l_{1}} \frac{P_{n}(x)-P_{n}(y)}{x-y} \varphi_{1}(y) d y+\int_{i_{1}} \frac{P_{n}(y) y_{1}(y) d y}{x-y}=R_{n}+\mu_{n},
\end{aligned}
$$

ed uguagliando separatamente in queste formole le parti intere in $x$ e le serie di potenze negative di $x$, si ottiene:

$$
\left.\begin{array}{l}
Q_{n}(x)=\int_{i} \frac{P_{n}(x)-P_{a}(y)}{x-y} \varphi(y) d y, \\
R_{n}(x)=\int_{l_{1}} \frac{P_{n}(x)-P_{n}(y)}{x-y} \varphi_{1}(y) d y,
\end{array}\right\}
$$


e

$$
\left.\begin{array}{l}
\lambda_{n}(x)=\int_{l} \frac{P_{n}(y) \circlearrowleft(y) d y}{x-y}, \\
\mu_{n}(x)=\int_{l_{1}} \frac{P_{n}(y) \varphi_{1}(y) d y}{x-y} \cdot
\end{array}\right\}
$$

Ma $\lambda_{n}, \mu_{n}$ sono dei gradi indicati al $\S 9$, onde risulta che:

$$
\begin{aligned}
& \int_{i} P_{n}(y) \varphi(y) y^{s} d y=0 \\
& \int_{i_{1}} P_{n}(y) \varphi_{1}(y) y^{s_{1}} d y==0
\end{aligned}
$$

per

$$
\left.\begin{array}{l}
s=0, \quad 1,2, \ldots r-1 \\
s_{1}=0, \quad 1,2, \ldots r-2
\end{array}\right\} \text { per } n=2 r,
$$

e per

$$
\begin{aligned}
& s=0, \quad 1,2, \ldots r-1 \\
& s_{1}=0, \quad 1,2, \ldots r-1
\end{aligned} ; \text { per } n=2 r+1
$$

\section{A PPLICAZIONI.}

I. Espressione dei coefficienti $a_{n}^{\prime}$ dell'algoritmo per mezzo delle funzioni $\varphi(y), \varphi_{1}(y)_{n}^{*}$ sotto il segno.

12. Nella teoria delle frazioni continue algebriche se una funzione:

$$
\sigma=\int_{i} \frac{\varphi(y) d y}{x-y}
$$

ammette lo sviluppo:

$$
\frac{1}{a_{1}^{\prime} x+a_{1}^{\prime \prime}-\cdots}, \ldots
$$


il coefficiente $a_{n}^{\prime}$ del quoziente incompleto $n$ simo ̀̀ dato dalla formola:

$$
\frac{1}{a^{\prime},}=\int_{l} P_{n}^{2}(y) \varphi(y) d y(*)
$$

o da altre in cui questa si può facilmente trasformare. Si domanda se per i coefficienti $a_{n 2}^{\prime}$ del presente algoritmo si possono dare formole analoghe.

A quest'effetto, deduciamo dalle (22), (23):

$$
\begin{aligned}
& \lambda_{n}=\sigma_{1} P_{n}-Q_{n}=-\frac{C_{n+1}}{P_{n+1}}-\frac{C_{n+2} P_{n}}{P_{n+1} P_{n+2}}-\frac{C_{n+3} I_{n}}{P_{n+2} P_{n+3}}-\cdots \\
& \mu_{n}=\sigma_{2} P_{n}-R_{n}=\frac{B_{n+1}}{P_{n+1}}+\frac{B_{n+2}}{P_{n+1} P_{n+2}}+\frac{B_{n+3}}{P_{n+2} P_{n+3}}+\cdots
\end{aligned}
$$

Il termine di più alto grado in $x$ viene dato in questi sviluppi dal primo termine, cioè rispettivamente dal termine di più alto grado in

$$
-\frac{C_{n+1}}{P_{n+1}} \text { e } \frac{B_{n+1}}{P_{n+1}}
$$

Si supponga prima $n=2 \vartheta+1$; il coefficiente del termine di più alto grado in $C_{n+1}$ ̀े $k_{2,+2}$, in $P_{n+1} \dot{\mathrm{e}}-h_{2++3} k_{2 r+2}$, onde per il coefficiente del termine di più alto grado in $-\frac{C_{n+1}}{P_{n+1}}$ cioè in $\lambda_{n}$ si trova il valore:

$$
\frac{1}{l_{2 r+3}}=\frac{1}{a_{0}^{\prime} a_{2}^{\prime} a_{4}^{\prime} \ldots a_{2 r}^{\prime}},
$$

in forza delle (14). Ma il coefficiente della più alta potenza di $x$ in $\lambda_{n}$ viene dato, in conseguenza delle $(26)$ e per essere $\lambda_{n}$ del grado $-(r+1)$, da:

$$
\int_{i} P_{2 x: 1}(y) \varphi(y) y^{2} d y
$$

onde:

$$
\frac{1}{a_{0}^{\prime} a_{2}^{\prime} a_{4}^{\prime} \ldots a_{2 r}}=\int_{i} P_{a r}(y) \varphi(y) y^{r} d y
$$

da cui si dedace:

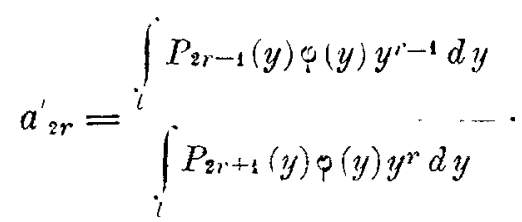

(*) Vedi jer es. Possé, Sur quelques applisatums des fractions continues algebriques, pag. J. S.t Pètersbourg, 1886. 
Si supponga ora $n=2 r$; il coeffeiente del termine di più alto grado di $B_{n-1}$ c̀ $h_{2 r+1}$, quello di $P_{n+1}$ è $h_{2 r+1} k_{2 r+2}$, onde quello di $\mu_{n}$ è, tenuto conto delle (14):

$$
\frac{1}{l_{2 r+2}}=\frac{1}{a_{1}^{\prime} a_{3}^{\prime} a_{5}^{\prime} \ldots a_{2 r-1}^{\prime}} .
$$

D'altra parte, essendo $\mu_{n}$ del grado $-r$, il coefficiente della più alta potenza di $x$ in $\mu_{n}$ è dato per le (26) da:

$$
\int_{i_{1}} P_{2 r}(y) \varphi_{1}(y) y^{r-1} d y
$$

onde:

$$
\frac{1}{a_{1} a_{3}^{\prime} a_{5}^{\prime} \ldots a_{21}-1}=\int_{l_{1}} P_{2 r}(y) \varphi_{1}(y) y^{r+1} d y
$$

ed infine:

$$
a_{2 r+1}^{\prime}=\frac{\int_{2 r}(y) O_{1}(y) y^{r-1} d y}{\int_{i_{1}} P_{2 r+1}(y) \varphi_{1}(y) y^{r} d y} .
$$

Le formole (29) e (31) rispondono alla questione posta in principio del presente paragrafo.

II. Rappresentazione simultanea (esatta od approssimata) di due integrali definiti mediante $n$ valori di una medesima funzione.

13. È facile mostrare come il nostro algoritmo sia atto a dare un'estensione del metodo classico di calcolo approssimato degl' integrali definiti, mediante $n$ valori di una stessa funzione. L'estensione consiste in ciò: che mentre il metodo detto dal Gauss delle quadrature meccaniche vale a risolvere la questione:

"Dato un integrale definito

$$
\int_{i} \varphi(y) \psi(y) d y
$$

"determinare $n$ punti $a_{1}, a_{2}, \ldots a_{n}$ e le costanti $c_{1}, c_{2}, \ldots c_{n}$ in modo tale " che l'integrale venga rappresentato colla massima possibile approssimazione " da un'espressione:

$$
c_{1} \psi\left(a_{1}\right)+c_{2} \psi\left(a_{2}\right)+\cdots+c_{n} \psi\left(a_{1}\right) ",
$$


invece col nostro metodo risolveremo la seguente questione, di apparenza più complessa:

"Dati due integrali defiriti:

$$
\int_{i} \varphi(y) \psi(y) d y, \quad \int_{i_{1}} \varphi_{1}(y) \psi(y) d y,
$$

“ determinare $n$ punti $a_{1}, a_{2}, \ldots a_{n}$ e le costanti $c_{1}, c_{2}, \ldots c_{n}$ e $c_{1}^{\prime}{ }_{1}, c_{2}^{\prime}, \ldots c_{n}^{\prime}$ " in modo tale che gl'integrali vengano rappresentati simultaneamente e colla “ massima possibile approssimazione da due espressioni della forma (32)."

14. A risolvere questo nuovo problema, si prenda dapprima un polinomio $F(y)$ razionale intero in $y$, di grado non maggiore di $3 r-1$, cssendo

$$
n=2 r+1 \text {. }
$$

La $P_{n}(y)$ è allora di grado $n-1=2 r$, e si può porre:

$$
F(y)=P_{n}(y) q(y)+r(y)
$$

dove il quoziente $q(y)$ è di grado $r-1$, jl resto $r(y)$ di grado non maggiore di $2 r-1$.

Supponendo per semplicità le radici $a_{*}$ di $P_{n}(y)$ distinte, e lasciando al lettore la facile modificazione delle formole che seguono nel caso che $P_{n}(y)$ abbia radici multiple, avremo dalla formola d'interpolazione di Lagranas:

$$
r(y)=P_{n}(y) \sum_{s=1}^{n-1} \frac{r\left(a_{n}\right)}{\left(y-a_{2}\right) P^{\prime}\left(a_{n}\right)}
$$

onde moltiplicando la (33) per $\varphi(y) d y$ ed integrando lungo $l$, si ha:

$$
\begin{gathered}
\int_{i} F(y) \Psi(y) d y=\int_{i} P_{n}(y) q(y) q(y) d y \\
+\sum_{y=1}^{n-1} \frac{r\left(a_{n}\right)}{P^{\prime}{ }_{n}\left(a_{i}\right)} \int_{i} \frac{P_{n}(y) \varphi(y) d y}{y-a} .
\end{gathered}
$$

Ma essendo $q(y)$ del grado $r-1$, risulta immediatamente dalle formole (27) che:

e dalle (21) e (26) che:

$$
\int_{i} P_{n}(y) q(y) \varphi(y) d y=0
$$

$$
\int_{i} \frac{P_{n}(y) \varphi(y) d y}{y-a_{y}}=-i_{n}\left(a_{n}\right)=Q_{n}\left(a_{n}\right)
$$


onde infine, essendo ancora $r\left(a_{v}\right)=F\left(a_{v}\right)$, viene:

$$
\int_{i} F(y) \varphi(y) d y=\sum_{\nu=1}^{n-1} \frac{F\left(a_{v}\right) Q_{n}\left(a_{v}\right)}{P_{n}^{\prime}\left(a_{v}\right)},
$$

ed indicando con $c_{v}$ le quantità :

$$
\frac{Q_{n}\left(a_{n}\right)}{P_{n}^{\prime}\left(a_{2}\right)}
$$

costanti indipendenti dalla speciale funzione $F(x)$ data, viene:

$$
\int_{i} F(y) \varphi(y) d y=\sum_{\nu=1}^{n-1} c_{\nu} F\left(a_{\nu}\right)
$$

Ma l'identico procedimento dà pure:

$$
\int_{\ell_{1}} F^{\prime}(y) \varphi_{1}(y) d y=\sum_{\nu=1}^{n-1} c_{\nu}^{\prime} F\left(a_{\nu}\right),
$$

con

$$
c^{\prime}{ }_{\nu}=\frac{R_{n}\left(a_{\nu}\right)}{P_{n}^{\prime}\left(a_{v}\right)}
$$

talchè le formole $(34),\left(34^{\prime}\right)$ ci dànno il seguente teorema:

"Se $F(y)$ è una funzione razionale intera di grado non maggiore di " $3 r-1$, gl' integrali:

$$
\int_{i} F(y) \varphi(y) d y, \quad \int_{l_{1}} F(y) \varphi_{1}(y) d y,
$$

" si possono esprimere mediante funzioni lineari a coeffcienti costanti di $F\left(a_{1}\right)$, " $F\left(a_{2}\right), \ldots F\left(a_{2}\right) . I$ coefficienti di queste espressioni sono indipendenti dalla "speciale $F(y)$, ed $i$ punti $a_{1}, a_{2}, \ldots a_{2 v}$ sono le radici del polinomio $P_{2 r+1}$, "ottenuto coll'applicazione del nostro algoritmo alle funzioni:

$$
\int_{i} \frac{\psi(y) d y}{x-y}, \quad \int_{l_{1}} \frac{\varphi_{1}(y) d y}{x-y} .
$$

15. Le formole (34) e (34'), che dànno un'espressione esatta per il caso in cui $F(y)$ è un polinomio razionale intero, servono invece come formole di 
approssimazione $\left({ }^{*}\right)$ per il caso che ad $F(y)$ si sostituisca una funzione analitica qualunque, rappresentata da una serie di potenze di $y$ intere e positive. Sia $\psi(y)$ questa serie, il cui cerchio di convergenza comprenda le linee d'integrazione $l$ ed $l_{i}$, e si ponga:

$$
\psi(y)=F(y)+\sum_{\mu=3 r}^{\infty} g_{\mu} y^{\mu} .
$$

essendo $F(y)$ l'insieme dei termini di grado $\leq 3 r-1$. Moltiplicando per $\varphi(y) d y$ ed integrando lungo $l$, poi moltiplicando per $\varphi_{1}(y) d y$ ed integrando lungo $l_{1}$, si avrà per le formole precedenti:

$$
\begin{aligned}
& \int_{l} \psi(y) \varphi(y) d y=\sum_{\nu=1}^{n-1} c_{\nu} F\left(a_{\nu}\right)+\sum_{\mu=3 r}^{\infty} g_{\mu} \int_{l} y^{\mu} \varphi(y) d y, \\
& \int_{i_{1}} \psi(y) \varphi_{1}(y) d y=\sum_{\nu=1}^{n-1} c_{\nu}{ }_{\nu} F\left(a_{\nu}\right)+\sum_{\mu=3 r}^{\infty} g_{\mu} \int_{l_{1}} y^{\mu_{\nu}} \varphi_{1}(y) d y,
\end{aligned}
$$

e ponendo per le $F\left(a_{*}\right)$ il loro valore tratto dalla $(35)$ :

$$
\begin{aligned}
& \int_{i} \psi(y) \varphi(y) d y=\sum_{\nu=1}^{n-1} c_{\nu} \psi\left(a_{\nu}\right)+\sum_{\nu=3 r}^{\infty} g_{\mu}\left\{\int_{i} y^{\mu} \varphi(y) d y-\sum_{\nu=1}^{n-1} a_{\nu}^{\mu}\right\}, \\
& \int_{l_{1}} \psi(y) \varphi_{1}(y) d y=\sum_{\nu=1}^{n-1} c_{\nu}^{\prime} \psi\left(a_{\nu}\right)+\sum_{p=3 r}^{\infty} g_{\mu}\left\{\int y^{\mu} \varphi_{1}(y) d y-\sum_{\nu=1}^{n-1} a_{\nu}^{\mu}\right\} .
\end{aligned}
$$

Da ciò segue che le espressioni lineari:

$$
\sum_{\nu=1}^{n-1} c_{\nu} \psi\left(a_{\nu}\right), \quad \sum_{\nu=1}^{n-1} c_{\nu}^{\prime} \psi\left(a_{\nu}\right)
$$

in cui $\mathrm{i}$ coefficienti e le $a_{*}$ sono indipendenti dalla speciale funzione $\psi(y)$, ma dipendono solo da $\varphi(y)$ e $\varphi_{1}(y)$, rappresentano approssimativamente gl'integrali del primo membro. Inoltre l'approssimazione, intesa nel debito senso, ̀̀ la massima possibile compatibilmente col numero delle indeterminate $a_{\nu}, c_{\nu}, c_{\nu}^{\prime}$ : infatti queste sono in numero di $6 \%$, c poichè i termini complementarj delle

(*) In ciò che segue, quando si parla di espressioni approssimate riferendosi ad una certa serie di potenza $\Sigma_{g}, x^{\nu}$, si deve intendere che nelle espressioni formate colle $g$, , si trascurano queste $g_{y}$ da un certo indice in avanti. In questo senso $\dot{e}$ intesa l'approssimazione nelle questioni di quallature meccoticlie ed affini. 
(36) non dipendono dai primi $3 r$ termini della serie $\psi(y)$, con esse si soddisfa appunto a $6 r$ condizioni.

Di più si noti che nei termini complementari i coefficienti $g_{3 r}, g_{3 r+1}, \ldots$ della $\psi(y)$ sono moltiplicati per quantità pure indipendenti dalla $\psi(y)$ stessa.

Se i limiti d'integrazione fossero reali, come pure le quantità $a_{v}$ e se queste fossero comprese fra i limiti d'integrazione, l'applicazione testuale di un metodo del MarkofF $\left(^{*}\right)$ fondato sull' uso di un notevole teorema di caicolo integrale dovuto all' Hermite $\left(^{* *}\right)$ permetterebbe di trovare due limiti fia i quali è compreso l'errore che si commette sostituendo le espressioni (37) agl' integrali definiti.

III. Rappresentazione (esatta o approssimata) della sommu di due integrali definiti mediante $n$ valori di due funzioni date.

16. Il nostro algoritmo si presta ancora alla applicazione esposta nei seguenti paragrafi, e che mi sembra non meno interessante della precedente. Cominciamo col sostituire nella (5) alle $\sigma_{1}$ e $\sigma_{2}$ le loro espressioni (24) in forma d'integrale definito, ed avremo:

$$
\sigma_{n}=A_{n}(x)+B_{n}(x) \int_{i} \frac{\varphi(y) d y}{x-y}+C_{n}(x) \int_{l_{1}} \frac{\varphi_{1}(y) d y}{x-y},
$$

donde con una trasformazione semplice e spesso usata si deduce:

$$
\sigma_{n}=\int_{l} \frac{B_{n}(y) \varphi(y) d y}{x-y}+\int_{l_{1}} \frac{C_{n}(y) \varphi_{1}(y) d y}{x-y}
$$

e

$$
A_{n}(x)=-\int_{i} \frac{B_{n}(x)-B_{\iota}(y)}{x-y} \varphi(y) d y-\int_{l_{1}} \frac{C_{n}(x)-C_{n}(y)}{x-y} \varphi_{1}(y) d y(* * *)
$$

Supponiamo ora, per fissare le idee, $n$ pari e eguale a $2 r(* * * *)$; allora $B_{n}$ e $C_{n}$ sono del grado $r-1$ come dallo specchio (8), $\sigma_{n}$ è del grado $-2 r$.

(*) Vedi Posś́, Opera citata, par. 76.

(**) Lettre it M. Borchardt. J, de Crello, tomo 84.

(***) Queste formole (38) e (39) sono un caso particolare di quelle date sotto i numeri (31) e (35) nella mia citata Memoria: Sagyio di una generalizzazione, ecc.

(**\%) La trattazione del caso di $n$ dispari procederebbe perfettamente analoga e si tralascia per brevità. 
Dalla (38) risulta:

$$
\int_{i} B_{n}(y) \varphi(y) y^{s} d y+\int_{\ell_{1}} C_{n}(y) \varphi_{1}(y) y^{s} d y=0
$$

per $s=0,1,2, \ldots 2 v-2$; per conseguenza se $h(y)$ è un polinomio razionale intero arbitrario d'ordine non maggiore di $2 v-2$, si avrà pure:

$$
\int_{i} B_{n}(y) \varphi(y) h(y) d y+\int_{i_{1}} C_{n}(y) \varphi_{1}(y) h(y) d y=0
$$

17. Ciò posto, siano $G(y)$ ed $H(y)$ due polinomii razionali interi d'oldine $\alpha$ non superiore a $3 r-3$ e non inferiore ad $r+1$, e poichè le considerazioni che seguono hanno importanza sopratutto per valori grandi di $r$, si può supporre senza restrizione $r>2$.

Si possono allora determinare tre polinomii razionali interi $q(y), G_{1}(y)$, $H_{1}(y)$, il primo di grado non maggiore di $2 r-2$, il secondo ed il terzo di grado $\beta$ inferiore ad $\alpha$, tali che sia identicamente:

$$
\begin{aligned}
& G(y)=B_{n}(y) q(y)+G_{1}(y), \\
& H(y)=C_{n}(y) q(y)+H_{1}(y) .
\end{aligned}
$$

Infatti questo sistema porta a $2 \alpha+2$ relazioni fra $\mathrm{i}$ coefficienti indeterminati di $q, H, H_{1}$, i quali sono in numero di $\alpha-r+2 \beta+4$; ma uno di questi potendosi fare uguale all'unità, per poter soddisfare al sistema deve essere:

$$
\alpha-v+2 \beta+3 \geqslant 2 \alpha+2 \text { con } \beta<\alpha,
$$

donde:

$$
\alpha>\beta \geq \frac{\alpha+r-1}{2}
$$

Ne viene che $\mathrm{i}$ due numeri $\alpha, \frac{\alpha+r-1}{2}$ devono dare:

$$
\alpha \geq \frac{x+r-1}{2}+1
$$

risultato che collima coll' jpotesi $\alpha \supseteq r+1$ già fatta su $\alpha$; le equazioni (41) si possono dunque sodảisfare.

Moltiplicando ora la prima di queste formole per $\varphi(y) d y$, la seconda 
per $\varphi_{1}(y) d y$, integrando lungo $l$ ed $l_{1}$ rispettivamente e sommando, si avrà:

$$
\int_{i} G(y) \varphi(y) d y+\int_{i_{1}} H(y) \varphi_{1}(y) d y=\int_{i} G_{1}(y) \varphi(y) d y+\int_{i_{1}} H_{1}(y) \varphi_{1}(y) d y
$$

essendo nulla la somma degl' integrali che contengono la $q(y)$, in forza della $\left(40^{\prime}\right)$.

La formola (42) ci dimostra dunque che l'espressione:

$$
S^{v}=\int_{i} G(y) \varphi(y) d y+\int_{i_{1}} H(y) \varphi_{1}(y) d y
$$

si può ricondurre ad un'espressione $S_{1}$ della stessa forma, in cui in luogo dei polinomii $G, H$, figurano i polinomii $G_{1}, H_{1}$ di grado inferiore.

Seguitando ad applicare questo procedimento, si finirà dunque col giungere ad un'espressione:

$$
S_{i}^{\prime}=\int_{i} G_{i}(y) \varphi(y) d y+\int_{i_{1}} H_{i}(y) \varphi_{1}(y) d y
$$

in cui il grado dei polinomii sarà $r$, se nella precedente era il minimo valore $r+1$ supposto per $\alpha$; ed oltre a questo punto non si potrà più applicare la riduzione. $\mathrm{Ma}$ si può scrivere:

$$
\left.\begin{array}{l}
G_{i}(y)=B_{n}(y)(y+a)+G_{i+1}(y), \\
H_{i}(y)=C_{n}(y)(y+b)+H_{i+1}(y)
\end{array}\right\}
$$

essendo $G_{i+1}, H_{i+1}$ del grado $r-1$ : infatti le relazioni da soddisfare per verificare le (43) sono $2 r+2$, e le quantità disponibili, cioè $a, b$ ed i coefficienti di $G_{i+1}$ ed $H_{i+1}$ sono pure $2 r+2$. Allora, tenuto conto della (40):

$$
S_{i}=a \int B_{n}(y) \varphi(y) d y+b \int_{l_{1}} C_{n}(y) \varphi_{1}(y) d y+S_{i+1},
$$

e posto:

$$
a \int_{l} B_{n}(y) \varphi(y) d y=c, \quad b \int_{l_{2}} C_{n}(y) \varphi_{1}(y) d y=c^{\prime}
$$

si ha:

$$
S=S_{i}=c+c^{\prime}+S_{i+1}
$$

Ora per la formola d'interpolazione di Lagrange, essendo $G_{i+1}, H_{i+1}$ del grado 
$v-1$ ed indicando con $\alpha_{\nu}$ le radici di $B_{n}(y)$ e con $\beta_{v}$ quelle di $C_{n}(y)$, (che si supporranno anche qui distinte, lasciando al lettore la facile generalizzazione) si ha:

$$
\begin{aligned}
& G_{i+1}(y)==B_{n}(y) \sum_{\nu=1}^{n-1} \frac{G_{i+1}\left(\alpha_{\nu}\right)}{\left(y-\alpha_{\nu}\right) B_{n}^{\prime}\left(\alpha_{\nu}\right)}, \\
& H_{i+1}(y)=C_{n}(y) \sum_{\nu=1}^{n-1} \frac{H_{i+1}\left(\beta_{v}\right)}{\left(y-\alpha_{\nu}\right) C_{n}^{\prime}\left(\beta_{\nu}\right)},
\end{aligned}
$$

onde:

$$
S_{i+1}=\sum_{\nu=1}^{n-1} c_{\nu} G_{i+1}\left(\alpha_{, j}\right)+\sum_{\nu=1}^{n-1} c_{\nu}^{\prime} H_{i+1}\left(\beta_{v}\right)
$$

posto:

$$
c_{\nu}=\int_{i} \frac{B_{n}(y) \varphi(y) d y}{y-\alpha_{\nu}}, \quad c^{\prime}{ }_{\nu}=\int_{\iota_{1}} \frac{C_{n}(y) \varphi_{1}(y) d y}{y-\alpha_{. \nu}} ;
$$

ma per le $(41), \ldots$ (43) segue che:

onde infine:

$$
G_{i+1}\left(\alpha_{v}\right)=G\left(\alpha_{v}\right), \quad H_{i+1}\left(\beta_{v}\right)=H\left(\beta_{y}\right),
$$

$$
\int_{i} G(y) \varphi(y) d y+\int_{i_{1}} H(y) \varphi_{1}(y) d y=c+c^{\prime}+\sum_{\nu=1}^{n-1}\left(c_{\nu} G\left(\alpha_{\nu}\right)+c_{\nu}^{\prime} H\left(\beta_{\nu}\right)\right),
$$

formola che esprime la somma dei due integrali mediante una somma di valori della funzione $G(y)$ in $r-1$ punti e della funzione $H(y)$ in $r-1$ altri punti convenientemente scelti.

È facile vedere che il problema risoluto dalla formola (44) è determinato. Essendo infatti $G$ del grado $3 r-3$, ed indicando con $R$ una funzione razionale intera di grado $r-1, \stackrel{G}{R}$ è esprimibile mediante la somma

$$
\sum_{i=1}^{n-1} \frac{G^{\prime}\left(\alpha_{v}\right)}{\left(y-\alpha_{v}\right) R^{\prime}\left(\alpha_{v}\right)}
$$

aumentata di un polinomio di grado $2 r-2$ in $y$ : moltjplicando per $\psi(y) R(y) d y$ ed integrando, viene una sommatoria della forma:

$$
\sum_{\nu=1}^{n-1} c_{\nu} G\left(\alpha_{\nu}\right)
$$

aumentata di una somma di $2 r-1$ nuove costanti, ed altrettante ne porta l'integrale relativo ad $H$. Ma di queste $4 r-2$ nuove costanti ne spariscono 
$4 r-4$ nella formola (44), che non ne conserva che la $c_{-}^{-} e$ la $c^{\prime}$; ed infatti le quantità che concorrono ad ottenere questo risultato sono le $c_{v}, c_{v}^{\prime}, \alpha_{\nu}, \beta_{v}$, in numero precisamente di $4 r-4$.

18. La formola precedente è esatta nel caso di due polinomii $G, H$ di grado non superiore a $3 r-3$. Se ora questi polinomii fossero di grado maggiore, o se ad essi si sostituissero due funzioni analitiche qualunque rappresentate dalle serie di potenze intere e positive $\psi(y), \psi_{1}(y)$, si otterrebbe la formola di approssimazione

$$
\int_{i} \psi(y) \varphi(y) d y+\int_{i_{1}} \psi_{1}(y) \varphi_{i}(y) d y=c+c^{\prime}+\sum_{\nu=1}^{n-1}\left(c_{\nu} \psi\left(\alpha_{\nu}\right)+c^{\prime}{ }_{\nu} \psi_{1}\left(\beta_{\nu}\right)\right)+\rho,
$$

in cui il termine complementare $\rho$ non dipende che dai coefficienti delle serie $\psi, \psi$, il cui indice è superiore a $3 r-3$. 